\title{
Who Are You Without Your Substance? Transforming Occupational Time Use in Recovery
}

\author{
Paula Jarrard, OTD ${ }^{1}$, Sadie Cunningham ${ }^{1}$, Paxton Granda ${ }^{1}$, Paige Harker ${ }^{1}$, Taylor Lannan ${ }^{1} \&$ Kristine Price $^{1}$ \\ ${ }^{1}$ Department of Applied Medicine and Rehabilitation, Occupational Therapy Program, Indiana State University, \\ Terre Haute, IN, United States \\ Correspondence: Paula Jarrard, Department of Applied Medicine and Rehabilitation, Indiana State University, N. \\ $5^{\text {th }}$ St., Terre Haute, IN 47809, United States.
}

Received: July 19, 2021

doi:10.5539/mas.v15n6p19
Accepted: October 13, 2021

Online Published: October 14, 2021

\begin{abstract}
Medically underserved rural communities struggle to meet challenging needs in response to the opioid crisis. The purpose of this study was to measure any benefit of an occupational therapy (OT) intervention group with participants in an addiction recovery program. Supervised OT graduate students implemented a five-week program at a faith-based non-profit organization in a small metro community. A weekly time management occupational-based intervention group based on the Action Over Inertia (AOI) manualized protocol focuses on motivating and providing strategies to successfully reintegrate individuals into the community by using meaningful activity and positively influencing levels of occupational balance and engagement (Krupa et al., 2003). Outcome measures included self-report of time use, occupational balance, occupational engagement, and goal identification, satisfaction, and performance. The need to effectively treat individuals with SUD is a public health priority. Results demonstrated positive outcomes with self-rating of time management, self-management skills, frequency of engaging in meaningful activities, and performance and satisfaction in meeting individual goals. This research adds to the limited evidence base in the OT literature for interdisciplinary treatment of this population using a manualized occupation-based intervention.
\end{abstract}

Keywords: Action Over Inertia, occupational therapy, opioid crisis, recovery, time use

\section{Introduction}

Substance use disorder (SUD) is an ever-growing issue worldwide with devastating impact on an individual's brain and behavior. According to the Substance Abuse and Mental Health Services Administration (SAMHSA, 2019), 6.8\% of the Indiana population was diagnosed with SUD between 2015 and 2017, a number that is just slightly below the national average of $7.5 \%$. The diagnosis of SUD, no longer denoted as addiction in the Diagnostic and Statistical Manual of Mental Disorders, $5^{\text {th }}$ Edition (DMS-V), is used to describe the "wide range of the disorder, from a mild form to a severe state of chronically relapsing, compulsive drug taking" (American Psychiatric Association, 2013). It presents as an inability to control the use of alcohol, a legal or illegal drug (e.g., marijuana, cocaine, hallucinogens, heroin, or inhalants), or a medication or psychotherapeutic such as prescription pain relievers, sedatives, tranquilizers, or stimulants (Mayo Foundation for Medical Education and Research, 2017; SAMHSA, 2019). In addition, SUD often co-occurs with multiple physical and mental conditions with a common variable of uncontrolled pain. Chronic pain interference was associated with a $41 \%$ relative increase in the risk of developing a prescription opioid use disorder. (Blanco et al., 2016). Of 87 percent of those who screened positive for illegal drug use, misuse of prescription drugs or heavy alcohol use suffered from chronic pain (Alford et al., 2016).

In addition to disruption in behavior and brain function, SUD negatively impacts body function, ability to participate in meaningful activities, and quality of life (QOLI) (Stoffel \& Moyers, 2004). When an individual is unable to perform the skills needed to function successfully, including but not limited to self-care, home and financial management, and fulfilling daily roles, treatment for dependency on substances can positively impact independence and well-being. In order to establish the most effective evidence-based treatments, a multidisciplinary team, including physician, psychiatry, psychology, nursing, occupational, physical, and recreational therapies, and social work, promotes a holistic, individualized approach. 
Occupational therapy (OT) services focus on self-care, leisure, and productivity through the use of structured, purposeful activities that emphasize the individual's strengths in pursuit of recovery (Buijsse et al., 1999). Individuals with SUD have shown patterns of pervasive and persistent imbalance and disengagement from daily occupations (Krupa et al., 2003; Leufstadius et al., 2004). For individuals diagnosed with SUD, personal and neurological factors interact with contextual influences such that seeking and using alcohol and other drugs become dominant occupations (American Psychiatric Association, 2000); therefore, treatment should focus on meaningful occupations and appropriate use of time that give the patient a sense of purpose. The Person-Environment-Occupation (PEO) model is a frequently used frame of reference in recovery treatments, with the focus being to create an optimal balance of its components (personal and neurological factors and their interaction with contextual influences) to achieve maximal occupational participation. Specifically OT's role in mental health is to improve participation in client-chosen meaningful activities, and provide opportunities for emotional fulfillment and personal growth (Krupa, 2003). Individuals with mental health conditions, including SUD, may benefit from support to establish and restore social and personal roles and utilize coping and self-management skills for daily activities to improve QOLI.

A review of literature does demonstrate limited evidence for the effectiveness of OT interventions in working with clients with SUD. About $75 \%$ of clients participating in a self-development group model rated "strongly agree" with satisfaction on general goals focused on developing coping skills, handling stressors, identifying and engaging in meaningful activities, self-management, and enhancing self-expression and communication. (Peloquin \& Ciro, 2013). Martin, Bliven \& Boisvert (2008) reported significant improvements in occupational performance, self-esteem and QOL following OT interventions within a substance recovery program. Outcomes of Occupational Performance History Interview (Version 2.0), Rosenberg Self-Esteem Scale, and the Quality of Life Rating Scale demonstrated marked improvements with significant differences and large effect sizes comparing intake and discharge data.

Two occupational therapy manualized intervention protocols have shown some promising results in working with clients. Let's Get Organized (LGO), focused on individuals with co-occurring emotional and Substance Use Disorders (White, 2007). LGO uses cognitive compensatory techniques such as maintaining an appointment book and using goal-directed, trial-and-error learning strategies as part of cognitive rehabilitation. LGO participants displayed improved time management, organization, planning skills, emotional regulation, and satisfaction with daily occupations. (Holmefur et al., 2019). Another generalized protocol, Lifestyle Redesign, was fashioned specifically to address prevention and management of chronic conditions, including pain management. Through development of healthy self-care habits and routines, including pain management, outcome measures including the Canadian Occupational Performance Measure (COPM), Brief Pain Inventory, and Pain Self-Efficacy Questionnaire demonstrated a positive effect on the QOLI, self-efficacy, and functional abilities of each individual (Simon, 2017).

Another manualized intervention protocol, Action Over Inertia (AOI) (Krupa et al., 2010), has been successfully used by OT practitioners to help guide interventions for individuals with serious mental illnesses by focusing on improving occupational balance and engagement (Edgelow \& Krupa, 2011). Occupational balance is defined as the organization of daily activities in a manner that enables health and well-being, allowing for variations in participation in labor and work, home management, parenting, leisure, and rest activities (Wilcock et al., 1997). Occupational engagement is defined as the importance of the individual's connection to and investment in occupations, as well as the degree to which occupations are grounded in the broader social context (Edgelow \& Krupa, 2011). The goal of this manualized program is to replace inappropriate habits brought on by serious mental illnesses, including SUD, by addressing the poor time use management and encouraging healthy behaviors for individuals. The AOI protocol strives to reintegrate individuals into the community by utilizing meaningful activity and positively influencing levels of occupational balance and engagement through education and group activities (Krupa et al., 2010). Time use, occupational balance, occupational engagement, and clinical utility are outcomes measured with use of the AOI program.

With few studies of low hierarchical evidence, the lack of a strong base to support efficacy in the provision of OT services in recovery treatment programs remains a common barrier for expansion of the role of OT in mental health. Rojo-Mota, Pedreo-Peres, and Huertas-Hoyas (2017) conducted a study to determine the validity and volume of research of OT intervention related specifically to treatment of individuals with SUD. It was confirmed that theoretical studies, professional role studies, qualitative studies and quantitative studies lack strong evidence to support OT services with SUD treatment. Much evidence does exist for OT interventions as part of psychiatric rehabilitation for community integration and supported employment and educational interventions (Noyes, Sololow, \& Arbesman, 2018). The Substance Abuse and Mental Health Services Administration (SAMHSA) 
designated licensed OT practitioners as one profession that states might utilize to be a part of a Certified Center for Behavioral Health Center (SAMHSA, 2015, 1 b.2). Currently however, only eight states have recognized OT practitioners as Qualified Mental Health Providers with more advocating to change licensure laws to address the need for expected growth in the demand for treating individuals with mental health and SUD (Wilburn, Hoss, Pudeler, Beukema, Rothernbuhler, \& Stoll, 2021).

Implementing a manualized program based on other validated OT interventions may contribute to the limited research and highlight the beneficial outcomes OT can have for individuals in recovery. Rojo-Mota et al. (2017) state despite establishment of conceptual frameworks acknowledging addiction itself as an occupation, there remains a critical need for the OT profession to evaluate treatments of individuals with SUD. It is the intent of this research to add to that body of evidence and to inform practitioners on use of the AOI program to support a transformation of occupational time use leading to a healthier lifestyle and quality of life.

The purpose of this study was to evaluate the outcomes of implementing an OT manualized group intervention, AOI, in an underserved community setting specializing in treatment of SUD. This faith-based site, certified by Indiana Affiliation of Recovery Residences (IARR), is an adult residential and outpatient facility providing substance abuse groups, Alcoholics Anonymous meetings, peer coaching, case management, life skills training, and mental health services. Most common addictions treated in this locale include methamphetamine and marijuana use. The AOI intervention protocol was adapted to meet the needs of this facility to coincide with current programming while incorporating additional restrictions posed by the ongoing COVID-19 pandemic. Preand post-outcome measures were compared to determine any benefit of intervention.

\section{Method}

\subsection{Participants}

After approval by the Institutional Review Board at the authors' institution, flyers advertising the study were distributed and posted in classrooms at the facility with information to contact the Principal Investigator. Participants were not assigned randomly, but were permitted to choose to be in the experimental or control group. Twenty participants provided informed consent with 15 volunteering for the experimental group and 5 volunteering for the control group. By the second week of the study, all members in the control group opted to switch to the experimental group. Of the 20 original participants, 14 were lost to follow-up due to transitioning out of the facility's program or relapse with transition to another provider. There was a total of six participants $(\mathrm{n}=6)$ who completed all pre- and post-assessments.

\subsection{Measures}

The Canadian Occupational Performance Measure (COPM) is a client-centered focused outcome measure using a structured interview format to rate performance, satisfaction, and the importance of completing one's activities of daily living (Law et al., 1991). The client rates specific self-care, leisure, and productivity activities based on a scale of one to ten $(1=$ not important; $10=$ extremely important). The client prioritizes five problem areas that are most important based on a scale of 1 to 10 ( $1=$ not important; $10=$ most important). The COPM provides the basis for creating client-centered goals that are meaningful for each individual. Much research supports its clinical use and acceptable ranges for internal consistency, test/retest reliability, validity, and ability to detect clinical changes over time (Law et al., 2021).

The Assessment of Time Management Skills (ATMS) is a self-report questionnaire designed to "assess awareness and use time management strategies to plan and manage life tasks" (White et al., 2013). ATMS is a 30-question survey assessing one's time management, planning, organizational, and preparation skills, as well as confidence level of using time management skills. Participants self-rate occupation-based activities on the Likert scale on a scale of 1 to 4 ( $1=$ none of the time; $4=$ all the time) with questions such as "I feel confident I can complete my daily routine" (White et al., 2013). The psychometric properties for ATMS show a strong validity measurement of $\mathrm{p}=0.001$, a good internal consistency reliability with $\alpha=0.86$, and test-retest reliability $\mathrm{r}=0.89$.

The Engagement in Meaningful Activities Survey (EMAS) is a self-report scale measuring a participant's engagement in meaningful occupations, with components involving personal values, competence and mastery in performing occupations, and meaningfulness of occupations within one's social group (Goldberg et al., 2002). Each of the 12 EMAS items begin with, "The activities I do..." and includes statements such as: "help me take care of myself," "reflect the kind of person I am,", "help me achieve something which gives me a sense of accomplishment," "give me a feeling of control," and "help me express my personal values." (Eakman, 2012). The psychometric properties for EMAS demonstrate a reliability of $\alpha=0.89$. Using a Likert scale of one to four, participants self-rate statements of 1 (rarely) to 4 (always) regarding activities that allow for further classification 
into categories of low (score $<29$ ), moderate (score $=29-41$ ), or high (score >41) meaningfulness. Eakman (2012) purports that engagement in meaningful occupations is a key outcome of occupational therapy services.

\subsection{Procedure}

Pre- and post-measures were collected prior to the first and after the final sessions. Consent form data were stored separately from outcome measure data and assigned random numerical codes to ensure confidentiality. Five weekly, 60-minute sessions were held virtually via Zoom per Covid-19 protocol restrictions, including a morning and evening time slot to accommodate all participants' schedules. Group sessions addressed occupational balance and time management skills to establish occupational routines supporting desired roles for successful community reintegration. Supervised OT research assistants implemented a weekly time management occupational-based intervention program following components of the AOI protocol (Table 1). Participants were issued a planner and set of colored fine tip markers to promote daily goal setting and apply time management skills.

Table 1. Intervention Based on Action Over Inertia Manual

\begin{tabular}{|c|c|c|}
\hline Week & Activities & AOI Chapter Reference \\
\hline 1 & Breaking plates icebreaker, understanding personal activity patterns & 2 \\
\hline 2 & Mason jar activity, making quick changes in personal activity patterns & 2,3 \\
\hline 3 & Healthy habits jeopardy: leisure, sleep/stress, nutrition, physical habits & 4 \\
\hline 4 & Identifying emotions/triggers and what healthy habits can help cope with barriers & 4 \\
\hline 5 & $\begin{array}{l}\text { Reflecting on daily time log/goals, making long term changes and supporting activity } \\
\text { change }\end{array}$ & 5,6 \\
\hline
\end{tabular}

\section{Results}

A descriptive experimental research design was used to analyze data from pre- and post-assessments (COPM) and self-report questionnaires (ATMS, EMAS). Once all the pre- and post-assessments were completed, scoring was compared to determine the effectiveness of the interventions. The research assistants translated results to analyze the outcomes to capture self-reflection of participants regarding their initial and concluding views on their satisfaction, performance, and participation in meaningful daily activities. Pre- and post-scores between all outcome measures were compared to determine personal participant improvements (Table 2).

\subsection{Canadian Occupational Performance Measure}

The COPM documented specific changes reported by participants regarding individual perceptions on goal satisfaction and performance. Based on the minimal clinically important difference (MCID) of 2 points, four participants significantly improved their performance and four significantly improved their satisfaction with desired goals. Individual goals were determined by each participant and could cover any domain in which they desired to improve. Some goals did not specifically address time management or life balance skills, but were more focused on achieving personal goals, such as increasing participation in work and parenting roles.

\subsection{Assessment of Time Management Skills}

The ATMS provided changes reported by the participants regarding their perceptions regarding individual time management skills. All six participants improved their scores. Changes ranged from an improvement of two up to 34 . Six of the 30 questions on this assessment relate specifically to planner use. Table 3 details these questions and change scores indicating overall benefit with a planner issued to each participant. Four of six participants utilized the provided planners as a time management strategy.

\subsection{Engagement in Meaningful Activities Survey}

The EMAS provided reported changes surrounding engagement in meaningful activities. Some individuals reported decreases in engagement in meaningful activities, however their COPM scores for performance and satisfaction had significantly increased. 
Table 2. Pre- and Post-Assessment Changes

\begin{tabular}{lllll}
\hline Participant & ATMS* & EMAS & COPM Performance** & COPM Satisfaction** \\
\hline 1 & 34 & 9 & $\mathbf{2}$ & $\mathbf{2 . 7 5}$ \\
2 & 16 & 3 & 0 & $\mathbf{2 . 3 3}$ \\
3 & 2 & -3 & $\mathbf{3 . 2 5}$ & $\mathbf{3 . 2 5}$ \\
4 & 4 & 6 & 0 & -0.33 \\
5 & 4 & 5 & $\mathbf{5}$ & 0.66 \\
6 & 12 & -6 & $\mathbf{6 . 3 3}$ & $\mathbf{6}$ \\
\hline
\end{tabular}

Note. ATMS = Assessment of Time Management Skills; EMAS = Engagement in Meaningful Activities Survey; COPM $=$ Canadian Occupational Performance Measure.

*Negative ATMS scores were transformed for analysis purposes

**Minimal Clinically Important Difference (MCID) for COPM $\geq 2$

Table 3. ATMS Change Scores Related to Use of Planner

\begin{tabular}{lllllll}
\hline & \multicolumn{7}{c}{ Participant Change Scores } \\
\hline ATMS Question & 1 & 2 & 3 & 4 & 5 & 6 \\
1.I feel I manage my time well & 2 & -2 & 0 & 1 & 1 & 1 \\
2. I use appointment book to remember daily tasks & 1 & 0 & -1 & -1 & 3 & 1 \\
3. I look at appointment book during day to keep track of my daily schedule & 1 & 1 & 0 & -2 & 2 & 0 \\
4. I carry an appointment book & 1 & 0 & 2 & 2 & 1 & 2 \\
6. I plan my daily activities & 1 & 0 & 1 & 0 & -1 & 2
\end{tabular}

\section{Discussion}

Occupational therapy is but one of several disciplines involved in the complex treatment of individuals with SUD. This pilot study featured a group intervention protocol based on an existing manualized program, Action Over Inertia, to determine its effectiveness in the setting of a residential faith-based facility in a small metro area serving many rural counties. All six participants rated time management and self-management skills improved as a result of the modified AOI protocol intervention. Four of the six participants utilized the provided planners as a time management strategy. All six participants demonstrated improved scores on the ATMS, indicating improvement in time management skills. Four of six participants rated the frequency of engaging in meaningful activities as increased based on comparison of EMAS scores.

Each client formulated personal goals which were rated objectively through assignment of numbers for occupational performance, satisfaction, and importance of each goal both pre- and post-intervention. Four of six participants met the minimal clinically important difference (MCID) in rating individualized performance and satisfaction as a result of the intervention. Interestingly, those participants whose total score indicated less frequent participation in meaningful activities (EMAS) had rated their individual goal performance and satisfaction on the COPM higher than other participants. One might speculate that these participants focused more on achieving their individual goals as opposed to restoring occupational balance with meaningful activities, still resulting in beneficial outcomes for those individuals.

The ongoing Covid-19 pandemic necessitated changes in the study design requiring group sessions to be held through Zoom platform rather than face to face at the facility. It remains unknown how this lack of face to face interaction impacted discussion and reflection in the process. Temporary technological failures contributed to some frustration to participants. Consequently, the positive outcomes can allow assumption that online intervention can in this case produce some effective changes.

Multiple factors pose limitations for interpreting efficacy of results of this study and determination of clinical utility across settings. Initially the study design was created to include both an experimental and control group. This may have permitted comparison for the efficacy of OT treatment with use of the modified AOI protocol versus usual treatment in this facility. However, it speaks to the positivity of the implemented interventions that participants were highly interested in the topics and wanted to transfer after the first session to the experimental group. Another limitation includes the small sample size of those who were able to participate in all sessions 
including pre- and post-data collection. Of 20 original participants, 14 were lost to follow-up with participants either transitioning out of the program or relapsing, contributing to a $70 \%$ dropout rate. Group sessions were implemented over a five-week period of time. COVID-19 protocol and need for quarantine during the time interval dictated that the groups received sessions via technology that was not always reliable. Research design did not provide for follow-up after the five-week group ended. These factors taken together certainly impact generalization of the findings of the current study.

Future research with a more robust design may be undertaken to demonstrate the effectiveness of using the AOI program with individuals with SUD. Ideally outcome measures from both randomly assigned experimental and control groups could be compared in multiple facilities that serve individuals with SUD. Designing a group that met more weeks versus fewer weeks might be a useful comparison for determining the optimal number of sessions required to show benefit. Following up of participants when the group concludes at a timed interval, such as three months out, might inform of any long-term benefits of the intervention. Further improvements can be made in future studies such as providing in person sessions to encourage social engagement and increased opportunities to process as a group. An opportunity to follow up with individuals upon relapse or transition out of the residential program may have provided more insight.

This study contributes to the limited evidence of OT intervention within SUD populations demonstrating benefit to recovery with focus on increasing occupational balance and occupational engagement. SUD continues to impact many individuals as communities strive to provide resources for this growing trend. Addiction programs offer varied services to aid in the recovery process to support re-establishment of meaningful occupational roles and successful community reintegration. A team approach may offer the most successful model to contribute to success of recovery programs. OT's can use their expertise and knowledge of daily occupations, time management skills, and positive coping strategies to support clients in restoring healthy habits and routines in the recovery process. Use of an existing manualized program, Action Over Inertia, demonstrated improvements in time management and self-management skills, both necessary to support long-term commitment to establishment of healthy routines required for successful recovery in individuals with SUD.

\section{Acknowledgements}

This study was supported by an Office of Sponsored Programs Graduate Research grant, at the authors' university.

We wish to acknowledge Next Step for providing participants for this study.

\section{References}

Alford, D., German, J., Samet, J., Cheng, D., Lloyd-Traveglini, C., \& Saitz, R. (2016). Primary care patients with drug use report chronic pain and self-medicate with alcohol and other drugs. Journal of General Internal Medicine, 31, 486-491. https://doi.org/10.1007/s11606-016-3586-5

American Psychiatric Association. (2013). Diagnostic and statistical manual of mental disorders (5th ed.). https://doi.org/10.1176/appi.books.9780890425596

Blanco, C., Wall, M., Okuda, M., Wang, S., Iza, M., \& Olfson, M. (2016). Pain as a predictor of opioid use disorder in a nationally representative sample. American Journal of Psychiatry, 173(12), 1189-1195. https://doi.org/10.1176/appi.ajp.2016.15091179

Buijsse, N., Caan, W., \& Davis, S. F. (1999). Occupational therapy in the treatment of addictive behaviours. British Journal of Therapy and Rehabilitation, 6, 300-307. https://doi.org/10.12968/bjtr.1999.6.6.13973

Eakman, A. (2012). Measurement characteristics of the Engagement in Meaningful Activities Survey in an age-diverse sample. American Journal of Occupational Therapy, 66(2), 20-29. https://doi.org/10.5014/ajot.2012.001867

Edgelow, M., \& Krupa, T. (2011). Randomized controlled pilot study of an occupational time-use intervention for people with serious mental illness. The American Journal of Occupational Therapy, 65(3), 267-276. https://doi.org/10.5014/ajot.2011.001313

Goldberg, B., Brintnell, E., \& Goldberg, J. (2002). Engagement in meaningful activities survey. PsycTESTS Dataset. https://doi.org/10.1037/t49908-000

Holmefur, M., Lidstrom-Holmqvist, K., Roshanay, A. H., Arvidsson, P., White, S., \& Janeslatt, G. (2019). Pilot study of Let's Get Organized: A group intervention for improving time management. American Journal of Occupational Therapy, 73(5). https://doi.org/10.5014/ajot.2019.032631 
Krupa, T., Edgelow, S., Chen, S., Mieras, C., Almas, A., Perry, A., Randloff-Gabriel, D., Jackson, J., \& Bransfield, M. (2010). Action Over Inertia: Addressing the activity-health needs of individuals with serious mental illness. Ottawa, Ontario: Canadian Association of Occupational Therapists.

Krupa, T., McLean, H., Eastabrook, S., Bonham, A., \& Baksh, L. (2003). Daily time use as a measure of community adjustment for persons served by assertive community treatment teams. American Journal of Occupational Therapy, 57, 558-565. https://doi.org/10.5014/ajot.57.5.558

Law, M., Baptiste, S., Carswell, A., McColl, M., Polatajko, H., \& Pollock, N. (1991). Canadian occupational performance measure. Toronto: $\mathrm{CAOT}=\mathrm{ACE}, 1991$

Law, M., Baptiste, S., Carswell, A., McColl, M., Polatajko, H., \& Pollock, N. (2021). Psychometric properties of the COPM. Retrieved from https://www.thecopm.ca/casestudy/psychometric-properties-of-the-copm/

Leufstadius, C., Erlandsson, L., \& Eklund, M. (2006). Time use and daily activities in people with persistent mental illness. Occupational Therapy International, 13, 123-141. https://doi.org/10.1002/oti.207

Martin, L. M., Bliven, M., \& Boisvert, R. (2008). Occupational performance, self-esteem, and quality of life in substance addictions recovery. OTJR: Occupation, Participation and Health, 28(2), 81-88. https://doi.org/10.3928/15394492-20080301-05

Mayo Foundation for Medical Education and Research. (2021). Drug addiction (Substance Use Disorder). Retrieved from https://www.mayoclinic.org/diseases-conditions/drug-addiction/symptoms-causes/syc-20365112

Minato, M., \& Zemke, R. (2004). Time use of people with schizophrenia living in the community. Occupational Therapy International, 11, 177-191. https://doi.org/10.1002/oti.205

Noyes, S., Sololow, H., \& Arbesman, M. (2018). Evidence for occupational therapy intervention with employment and education for adults with serious mental illness: A systematic review. American Journal of Occupational Therapy, 72, 7205190010. https://doi.org/10.5014/ajot.2018.033068

Peloquin, S., \& Ciro, C. (2013). Self-development groups among women in recovery: Client perceptions of satisfaction and engagement. The American Journal of Occupational Therapy, 67(1), 82-90. https://doi.org/10.5014/ajot.2013.004796

Rockville, MD: Substance Abuse and Mental Health Services Administration. Retrieved from https://store.samhsa.gov/product/Behavioral-Health-Barometer-Volume-5/sma19-Baro-17-US

Rojo-Mota, G., Pedrero-Perez, E., \& Huertas-Hoyas, E. (2017). Centennial topics-Systematic review of occupational therapy in the treatment of addiction: Models, practice and qualitative and quantitative research. American Journal of Occupational Therapy, 71. https://doi.org/10.5014/ajot.2017.022061

Simon, A., \& Collins, C. (2017). Lifestyle redesign for chronic pain management: A retrospective clinical efficacy study. The American Journal of Occupational Therapy, 71. https://doi.org/10.5014/ajot.2017.025502

Stoffel, V., \& Moyers, P. (2004). An evidence-based and occupational perspective of interventions for persons with substance-use disorders. The American Journal of Occupational Therapy, 58, 570-586. https://doi.org/10.5014/ajot.58.5.570

Substance Abuse and Mental Health Services Administration. (2019). Behavioral Health Barometer: Indiana, Volume 5. Indicators as measured through the 2017 National Survey on Drug Use and Health and the National Survey of Substance Abuse Treatment Services.

Substance Abuse Mental Health Services Administration (SAMHSA). (2015). Criteria for the demonstration program to improve community mental health centers and to establish Certified Community Behavioral Health Clinics. Retrieved from https://www.samhsa.gov/sites/default/files/programs_campaigns/ccbhc-criteria.pdf

White, S. (2007). Let's Get Organized: An intervention for persons with co-occurring disorders. Psychiatric Services, 58(5), 713. https://doi.org/10.1176/ps.2007.58.5.713

White, S., Riley, A., \& Flom, P. (2013). Assessment of time management skills (ATMS): A practice-based outcome questionnaire. Occupational Therapy in Mental Health, 29(3), 215-231. https://doi.org/10.1080/0164212X.2013.819481 
Wilburn, V., Hoss, A., Pudeler, M., Beukema, E., Rothernbuhler, C., \& Stoll, H. (2021). Receiving recognition: A case for occupational therapy practitioners as mental and behavioral health providers. American Journal of Occupational Therapy, 75(5). https://doi.org/10.5014/ajot.2021.044727

Wilcock, A., Chelin, M., Hall, M., Hamley, N., Morrison, B., \& Scrivener, L. (1997). The relationship between occupational balance and health: A pilot study. Occupational Therapy International, 4, 17-30. https://doi.org/10.1002/oti.45

\section{Copyrights}

Copyright for this article is retained by the author(s), with first publication rights granted to the journal.

This is an open-access article distributed under the terms and conditions of the Creative Commons Attribution license (http://creativecommons.org/licenses/by/4.0/). 